
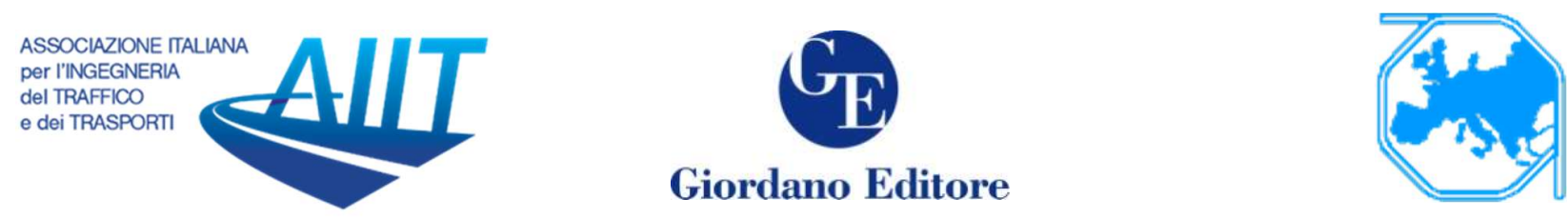

\title{
Development of a Microscopic Fuzzy Inference Based Cellular Automata Model for Pedestrian Flow
}

\author{
Ujjal Chattaraj ${ }^{1 *}$
}

${ }^{1}$ National Institute of Technology Rourkela

\begin{abstract}
In this work a microscopic model on pedestrian flow has been proposed. Observed data is used to calibrate and validate the proposed model. The model developed here uses a fuzzy inference system to represent the rules and a force-field structure to represent the repulsive and attractive impacts of objects and goals, respectively in the flow space. The flow space and time are discretized and viewed as discrete quantities. This microscopic model of pedestrian behavior, which essentially models how each pedestrian behaves over time in the flow space, is embedded in a simulation model which is used to simulate situations similar to the ones for which experiments have been done. The results show that the model performs reasonably well.
\end{abstract}

Keywords: microscopic, discrete, cellular automata, fuzzy inference system.

\section{Introduction}

The most basic mode of transportation is walking. Even with large studies made in developing faster (and safe) modes of transportation, walking remains an integral part of any trip as it continues to be a mode which can provide very high levels of accessibility to different facilities. It goes without saying that in order to better design spaces provided for human circulation (for example, in airports, shopping malls, fair grounds or even side- walks) one needs to study pedestrian motion empirically and develop models for the same. In this present study an attempt is made to develop a model for pedestrian behavior. The developed model is focused to describe pedestrian motion broadly on movement through corridors.

The model developed here assumes that pedestrians have a set of simple rules which they evaluate, may be subconsciously, while moving in a space. The rules are assumed to arise from a pedestrian's desire to reach the destination quickly and without bumping into obstacles or other pedestrians. The space in which a pedestrian moves contains both static and dynamic objects. The static objects are either static obstacles in the flow space which typically repel pedestrians from them or static goals (like egress points) which attract pedestrians. The dynamic objects are other pedestrians who, it is assumed, have a dual impact. On one hand they attract other pedestrians as they can act as pathfinders while on the other hand they repel as they are also physical objects not to be collided with. In this study, an attempt is made to model pedestrian dynamics by trying to

\footnotetext{
* Corresponding author: Ujjal Chattaraj (chattaraju@nitrkl.ac.in)
} 
understand and mimic the thought process employed by humans while moving.

In this study, the flow space is assumed to be occupied by static features (goals and obstacles) and dynamic features (other pedestrians). Each static feature is assumed to create attractive (for goals) or repulsive (for obstacles) potential fields (similar to force field model). Pedestrians are assumed to get influenced by dynamic features also. The dynamic features are the other pedestrians or groups of pedestrians. While moving a pedestrian implicitly decides, at every instant of time, the direction in which he/she should move and at what speed.

This paper is divided into five sections of which this is the first. The second section presents a review of the past work done on existing models of pedestrian behaviour. Section 3 describes in detail the proposed model for pedestrian motion. This section first describes how time and space is viewed in the model. Next, it concentrates on describing how static objects are modelled followed by a discussion on dynamic objects (other pedestrians) and how they are modelled. Later the structure of the fuzzy inference system used in the proposed model and how the proposed model is embedded in a pedestrian flow simulation model are described. Section 4 presents the results obtained from the proposed model. These results are used to validate the model comparing with the observed data obtained by conducting experiments. Section 5 concludes the paper by highlighting the contributions of this study as well as its shortcomings.

\section{Literature Review}

Understanding and modelling pedestrian movements, especially, at reasonable to high densities is important for design of any facility which involves pedestrians. In this section a review is presented on past work related to pedestrian flow modeling, followed by the motivation behind this work.

Existing models in literature can be categorized into two groups, macroscopic models and microscopic models. Macroscopic models are concerned with the overall behavior of the entire pedestrian stream (or crowd); whereas, microscopic models consider the decision making of individual pedestrian, which when agglomerated can give explanation of the stream/crowd behavior. So, in this paper a microscopic model is proposed. Keeping this fact in mind, in this section literature on existing microscopic models are discussed with more emphasis.

Microscopic models can be broadly classified into: (i) force based models and (ii) decision based models. Force based models typically work with a continuous representation of space and time while decision based models work with a discrete representation of space and time.

Force based models assume that pedestrians move due to an interplay between various attractive and repulsive forces which they face. These forces emanate from different goals and obstacles (like other pedestrians) which are present in the flow space. The different types of models differ in the way they represent these forces.

Most prominent in this group are different variants of Social Force Model (Helbing and Molnar (1995), Helbing (1991), Helbing (2000), Helbing et al. (2002), Mehran et al. (2009), Song et al. (2006)), which consider two types of forces controlling pedestrian flow. One is an attractive force due to goals or destinations. The other is a repulsive force due to obstacles like strangers, walls, streets, etc. In this group of model it is hypothesized that behavior of pedestrians can be determined by their intended velocity, effects of several attractive and repulsive factors and some fluctuations. Social Force Model has been applied to microsimulation of pedestrian traffic to explain accessibility and walkability for the development of sustainable mobility (Gruden et al. (2019), Habib et al. (2021), Amprasi et al. (2020)). 
Some other models in this group are Magnetic Force Model (Okazaki and Matsushita (1993)) and Centrifugal Force Model (Yu et al. (2005)).

Rule based models consider both space and time as discrete, leading to ease of computation. All of these models use some rule sets which control the motion of pedestrians.

Most prominent rule based model is Cellular Automata Model. Here, the whole pedestrian flow space is divided into a grid of cells and time is divided into discrete time steps. Movement from one cell to another occurs depending on some pre-defined rules and availability of empty cells; in these models a cell is assumed to be occupied by only one person at a time. Various form of cellular automata models have been proposed for various flow conditions, like, unidirectional flow (Blue and Adler (1998)), bi-directional flow (Blue and Adler (1999)) and cross-directional flow (Blue and Adler (2000)).

Other rule based models are Lattice Gas Model (Rothman and Zaleski (1994)), Mean Field Model (Nagatani (2001)), Benefit Cost Model (Gipps and Marksjoe (1985)) and Radial Grid Model (Antonini and Bierlaire (2006)).

It is to be mentioned here that among the existing models on pedestrian flow, force based models, which involve implicit formula, require high computational cost and are often unable to simulate movements of large number of pedestrians; whereas, the available decision based models (including the probability-based lattice gas models) are based on ad-hoc rule sets and are typically applicable to specific situations. Also, the existing models do not adequately address the imprecise nature of human decision making. Thus a pedestrian flow model with reduced computational cost which attempts to take into account the imprecise human decision making process is proposed in the present work.

\section{Proposed Model}

The proposed model is developed in a cellular framework having square cells of size $0.4 \mathrm{~m}$ (approximate body size of human being), using parallel update scheme (updating time is 0.5 second).

In the flow space a pedestrian takes two kinds of decisions while moving, namely, choice of direction and choice of speed. In order to take these decisions, he/she perceives the environment (static and dynamic features), utilizes his/her experience and commonsense to infer a course of action and responds (in terms of movement).

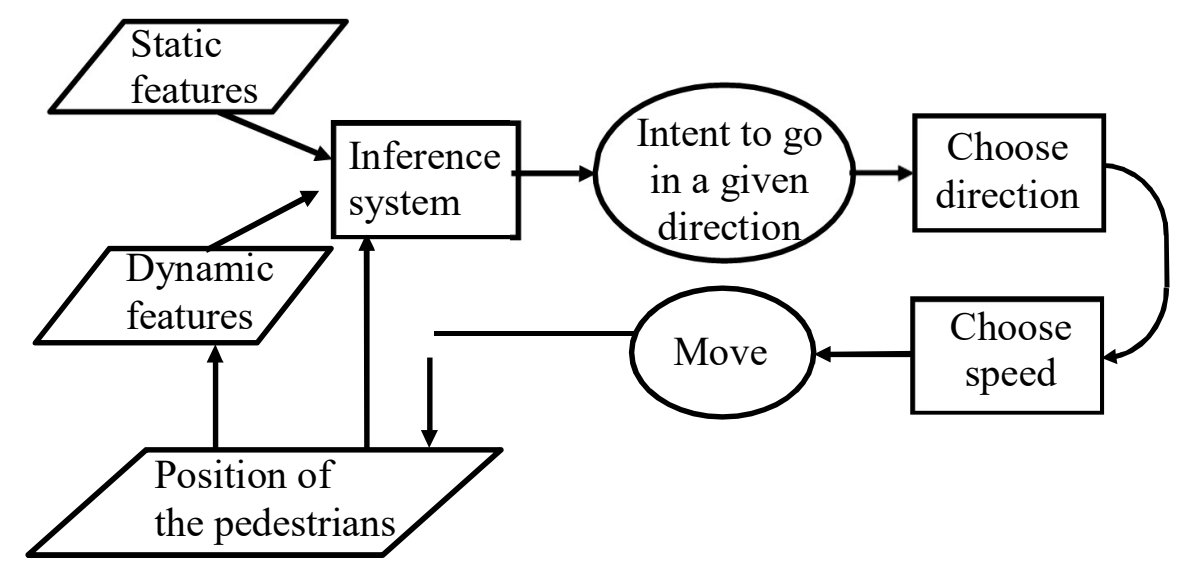


Figure 1: Structure of the model

The basic structure of the model developed here is shown in Figure 1. The schematic shown in Figure 1 is of an iterative process, which terminates at the end of the simulation period. The inference system takes input in the form of perceived static and dynamic features of the flowspace and develops a partial order among the possible directions in which the pedestrian can move. This information is utilized to choose a direction as well as an appropriate speed with which the person eventually moves. This movement and movement of others (each of whom, it is assumed, go through a similar process) impacts the dynamic features of the flow space and the process goes on over time. The inference system used here is a fuzzy inference system wherein the world view and the decision making processes are assumed to be approximate. In the foregoing part of this section the model is detailed.

\subsection{Static features}

The position, shape and size of static features remain same throughout. Goals increase attractiveness of a path; whereas, obstacles reduce attractiveness of the path. The attractiveness (or repulsiveness) of these features are represented as a potential field. As the goals are sources of attraction, they are assumed to emanate negative potential from themselves. On the contrary, as obstacles pose hindrance to movement their effect is represented as a positive potential, which repels people as they approach them.

\subsection{Dynamic features}

The only dynamic feature considered here is pedestrians or groups ofpedestrians. Specifically, two aspects of dynamic features are looked at; namely, the size and the average group velocity of a crowd. Here, these two properties are named as, "crowd level" and "crowd movement," respectively. Before describing them, the term, zone, is introduced. A zone is a specified area in the flow space defined with respect to a moving person. For example, for a given person the zones can be "ahead," "to the right," etc. Obviously, these will be different for different pedestrians; that is, at a given time the cells constituting the zones "ahead" for two different persons will be different because the persons' locations and orientations are different.

\subsubsection{Crowd level}

Let the number of pedestrians in a zone $z$ be nop $z$ and let the number of cells in that zone be $\operatorname{noc}_{\mathrm{z}}$. The crowd level of zone $z, \mathrm{cl}_{\mathrm{z}}$, is defined as

$$
\mathrm{cl}_{\mathrm{z}}=\frac{\left(\frac{n o p_{Z}}{n o c_{Z}}\right)}{\sum_{Z=1}^{Z}\left(\frac{n o p_{Z}}{n o c_{Z}}\right)}
$$

Where $Z$ is the total number of zones for a given person.

\subsubsection{Crowd movement}

In Figure 2, zone $A$ is marked as an arbitrary zone for pedestrian $p$ currently located as shown (It is to be mentioned here that for the purpose of simplicity the cellular flow space in Figure 2 is represented by continuous lines). Consider a person ' $\mathrm{k}$ ' in zone $A$ facing in a direction referred to as "face direction." The line marked "representative direction" is a direction which zone $A$ is supposed to indicate, like "ahead" or "to the right," etc. 
The crowd movement is defined as a speed representing the progress of the crowd in the representative direction. Hence $\mathrm{cm}_{\mathrm{z}}$, crowd movement in zone $z$ is defined as:

$$
\mathrm{cm}_{\mathrm{z}}=\frac{\Sigma_{\forall k \in P_{z}} v_{k} \cdot \cos k}{|P z| \cdot v^{\max }}
$$

Where,

$P_{z}$ is the set of pedestrians in zone $z$,

$v_{k}$ : speed of the $\mathrm{k}^{\text {th }}$ pedestrian in zone $z$ in the "face direction,"

$|\mathrm{Pz}|$ is the cardinality of set $\mathrm{Pz}$ (note $|\mathrm{Pz}|=$ nop $_{z}$ )

and $v^{\max }$ is the maximum speed at which pedestrians can move.

Note that $\mathrm{cl}_{\mathrm{z}}$ and $\mathrm{cm}_{\mathrm{z}}$ are different for different pedestrians and change with time.

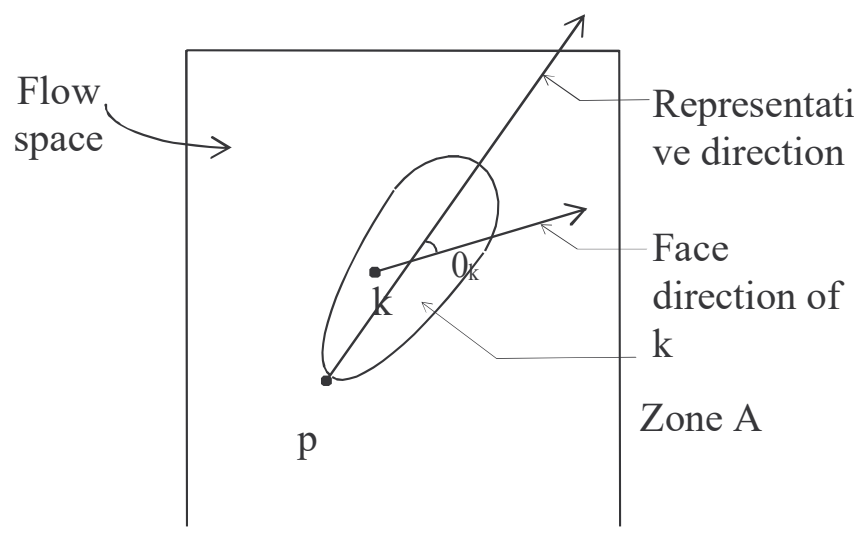

Figure 2: Zone surrounding a representative direction

\subsection{Inference system}

Lying at the heart of the model is an inference system to determine the direction in which a pedestrian would "like" to move at a given point in time. A fuzzy inference system is used since it is felt that the factors crowd level, crowd movement, effect of goals and obstacles are perceived by humans only approximately. The method used to construct the fuzzy inference rules can be viewed from Chattaraj and Subhashini (2015) and Chattaraj and Garnaik (2015).

\subsubsection{Premise variables (Antecedents)}

In the proposed fuzzy inference system three premise variables, namely, Attractiveness of path, Crowd level and Crowd movement are used as evaluators of a given direction. This is done because it is assumed that a pedestrian while considering a direction sees (i) whether there are obstacles in that direction or whether that direction will take them towards their goal (this is sought to be captured by the factor "attractiveness of path,") (ii) whether a given direction is crowded or not 
(captured through the factor "crowd level,") and (iii) whether, if there are some people in a given direction, the people are moving in a way which is advantageous to the pedestrian (captured through the factor "crowd movement.")

The three premise variables are further described with regard to their linguistic classifications in the following:

(a) Attractiveness of a path or direction: This seeks to capture how useful/good a path is to the pedestrian, irrespective of the number of pedestrians on that path. This is measured by $a_{z}$ the average of the maximum and minimum potential among all the cells in the direction being evaluated within a certain pre-determined distance. With decrease in average potential, the attractiveness of a path increases.

The Attractiveness of path is divided into three linguistic classes, namely, highly attractive, moderately attractive and not attractive. The three linguistic classes are described as fuzzy sets defined on $a_{z}$. Three membership functions for three sets are defined. The membership function $\mu_{\text {hap }}\left(\mathrm{a}_{\mathrm{z}}\right)$, describing the set "Highly attractive path" is described as

$$
\text { uhap }\left(a_{z}\right)=\left\{\begin{array}{c}
1 ; a_{z} \leq a_{z}^{1} \\
\frac{a_{z}^{2}-a_{z}}{a_{z}^{2}-a \frac{1}{z}} a_{z}^{1}<a_{z} \leq a_{z}^{2} \\
0 ; a_{z}>a_{z}^{2}
\end{array}\right.
$$

Similarly, $\mu_{\text {map }}\left(a_{z}\right)$ and $\mu_{\text {nap }}\left(a_{z}\right)$ describe membership functions of the sets "Moderately attractive path" and "Not attractive path," respectively. Figure 3 shows these membership functions.

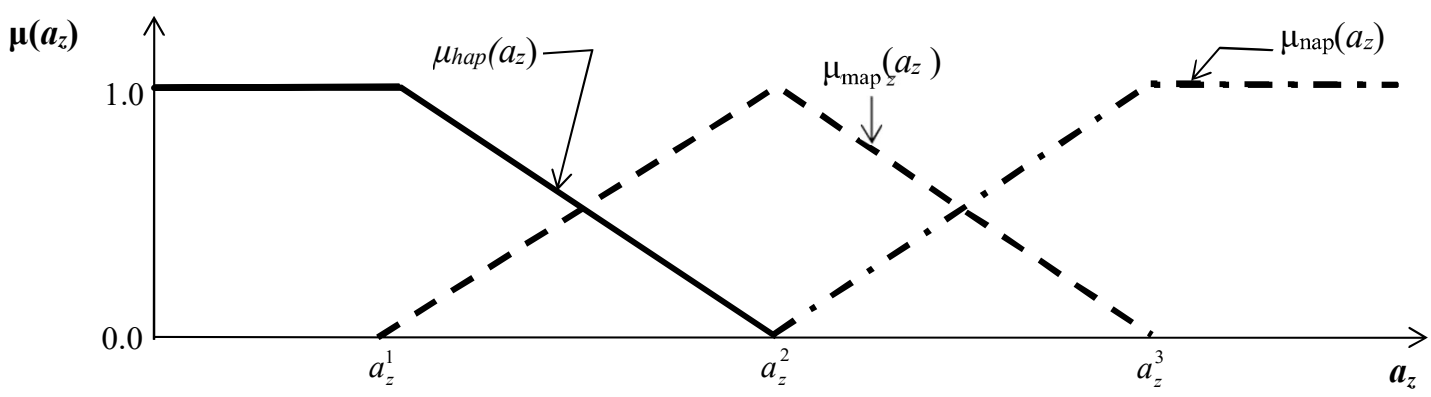

Figure 3: Membership functions for sets on Attractiveness of a path

(b) Crowd level: This seeks to capture how big the crowd is in a given direction. This is measured by $\mathrm{Cl}_{z}$ as described earlier. The Crowd level is divided into three linguistic classes, namely, negligible, moderate and large. Their membership functions are de- scribed by $\mu_{\mathrm{n}}\left(\mathrm{cl}_{\mathrm{z}}\right), \mu_{\mathrm{m}}\left(\mathrm{cl}_{\mathrm{z}}\right)$, and $\mu_{1}\left(\mathrm{cl}_{\mathrm{z}}\right)$ in a similar way as Attractiveness of path. 
(c) Crowd Movement: This seeks to capture how well the group of pedestrians in a given direction are moving. This is measured by $\mathrm{cm}_{z}$ as described earlier. The Crowd movement is divided into three linguistic classes, namely, bad, non-committal and good. Their membership functions are described by $\mu_{\mathrm{b}}\left(\mathrm{cm}_{\mathrm{z}}\right), \mu_{\mathrm{nc}}\left(\mathrm{cm}_{\mathrm{z}}\right)$, and $\mu_{\mathrm{g}}\left(\mathrm{cm}_{\mathrm{z}}\right)$ in a similar way as Attractiveness of path.

\subsubsection{Consequence variable}

The consequence variable in this fuzzy inference system represents the intent of a pedestrian to move along the path being evaluated. It is represented on a scale of -1 to 1 ; the scale is named "intent-to-go" or ITG scale. The variable describing the ITG scale is referred to as $\rho$; i.e., $\rho[-1$, 1]. A $\rho$ value of -1 on the ITG scale indicates "Avoid with absolute confidence," a +1 indicates "Go with absolute confidence," whereas, a 0 indicates complete indecision or confusion as to whether to go or not along that path.

\subsubsection{Rules of the fuzzy inference system}

The fuzzy inference system developed here relates the premise variables to the consequence variable, ITG through a set of rules. In this inference system there are in all 27 rules each relating a particular combination of the premise variables to a $\rho$ value; for example,

\section{If Attractiveness of path is highly attractive AND \\ Crowd level is negligible AND \\ Crowd movement is good Then}

\section{Go with high confidence}

The rules are developed based on the following principles:

1. $\rho$ increases as the attractiveness of a path increases,

2. $\rho$ is low when crowd level is large and high when it is moderate, and

3. $\rho$ increases as crowd movement becomes better.

Based on these basic principles a mapping is developed between the various combinations of fuzzy sets describing the premise variables and the ITG scale.

Figure 4 shows a schematic of such a mapping. In order to maintain clarity in the figure mapping for all the rules are not shown in the figure; a few are shown to indicate how $\rho$ values are chosen for the 27 rules.

\subsection{Choice of direction and speed}

For any given direction (zone) $z$, the $\mathrm{a}_{\mathrm{z}}, \mathrm{cl}_{\mathrm{z}}$ and $\mathrm{cm}_{\mathrm{z}}$ are known. These values are then used as input for the fuzzy inference system and the value of $\rho$ corresponding to these input values are determined. The value of $\rho$ for each direction is then used as an input for choosing a direction for movement of pedestrian ' $p$ ' at time $t$ using the following algorithm.

In this algorithm the current direction of the pedestrian is called the face direction, $f$ and the $\rho$ value for this direction as $\rho_{f}$.

1. Compare all possible directions (or zones) to determine that direction which has the highest $\rho$ value. Name this direction as best direction, $b$ and the corresponding $\rho$ as $\rho_{b}$ 


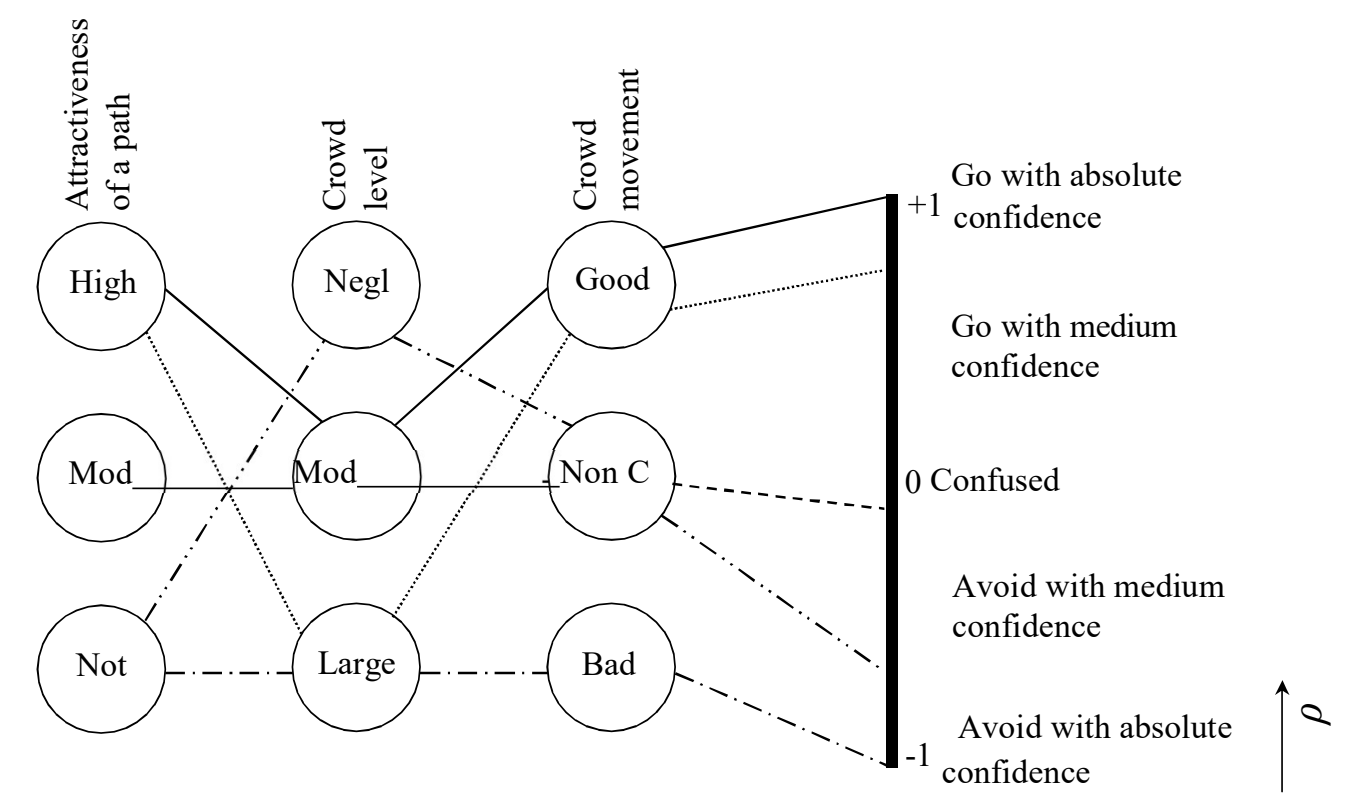

Figure 4: Mapping between premise variables and the ITG (or $\rho$ ) scale

2. (a) Let $\mathrm{c}$ be the chosen direction; $\mathrm{c}$ is given as:

$$
\mathrm{c}=\left\{\begin{array}{c}
\text { face direction, } f ; \text { if } \rho b-\rho f \leq T 1 \\
\text { best direction, } b ; \text { if } \rho b-\rho f \geq T 2 \\
\text { See Step } 2(b) ; \text { otherwise }
\end{array}\right.
$$

Here, $\mathrm{T}_{1}$ and $\mathrm{T}_{2}$ are the lower and upper threshold values, which indicate (i) when a pedestrian thinks it is not worthwhile to change directions, and (ii) when he/she thinks it is definitely worthwhile to change, respectively.

(b) When, $\mathrm{T}_{1}<\rho_{b}-\rho_{f}<\mathrm{T}_{2}$ it is assumed that a pedestrian is confused as to whether he/she should change. In such a case the following steps are used.

$$
\mathrm{c}=\left\{\begin{array}{c}
f ; \text { if gap in direction } b \text { is zero and gap in direction } f \text { is not zero } \\
b ; \text { if gap in direction } f \text { is zero and gap in direction } b \text { is not zero } \\
\text { See Step } 2(c) \text {; otherwise }
\end{array}\right.
$$

(c) Choose direction $b$ with probability $\frac{\rho b \quad \rho f}{T 2-T 1}$

3. (a) If the chosen direction $\mathrm{c}$ has gap equal to zero wait facing the direction $\mathrm{c}$ for 1 time step.

(b) If in the next time step gap in direction $\mathrm{c}$ is still zero; remove direction $\mathrm{c}$ and all other directions with zero gap from the list of possible directions. If the set of directions remaining is a 
null set continue to wait facing c else repeat steps 1 to 2 to determine a different $\mathrm{c}$.

After a direction $\mathrm{c}$ is chosen for movement, the next task is to choose the comfortable speed in the chosen direction. It is assumed that, in one time-step a pedestrian $p$ chooses to move in the chosen direction $\mathrm{c}$ by an amount Min $\left(\operatorname{gap}_{p c}, u^{d}\right)$; where, $u^{d}$ is the desired speed of pedestrian $p$ in units of distance per time-step. Here, it is assumed that, the "gappc" is not the actual gap (or distance) that exists, but rather a perceived gap. It is further assumed that this perceived gap for pedestrian $p$ in the direction $\mathrm{c}$ is given by,

$$
\operatorname{gap}_{p c}=\operatorname{Pgap}_{p c}-B_{p}
$$

where, $\operatorname{Pgap}_{p c}$ is the actual physical gap in front of pedestrian $p$ in direction c and $B_{p}$ is the buffer for pedestrian $p$ and is given by

$$
B=c_{1}+c_{2} \cdot u
$$

In Equation 4, $u$ is the current speed of the pedestrian $p ; c_{1}$ is a parameter which defines the buffer a pedestrian wants to keep in stopped condition and $c_{2}$ defines how this changes with speed. In some sense both $c_{1}$ and $c_{2}$ indicate a pedestrian's notion of "personal space" (or aversion to collision) while walking with others.

\subsection{Movement}

After a suitable direction and a suitable speed is chosen pedestrian $p$ takes the action to move. All the pedestrians in the flow space move simultaneously, i.e., parallel update scheme is adapted. Since more than one pedestrian cannot occupy a single cell simultaneously, if more than one pedestrian compete for the same cell at a time, tie is broken randomly. The winner in tie occupies the target cell and the others are made to move by the amount 1 cell lesser than their intended movement. After all the pedestrians are moved simultaneously with their respective speeds, the dynamic features change. Thus, at every time step the dynamic features of the input conditions to the fuzzy inference system change; whereas, the static features remain unchanged.

Since this developed pedestrian dynamics model is based on fuzzy inference and the movements of entities occur on cellular domain it is named as FICAPeD, acronym for Fuzzy Inference based Cellular Automata model for Pedestrian Dynamics.

All the model parameters are calibrated through trial and error using half of the experimentally observed data. The remaining half of the experimentally observed data are used to validate the model by comparing with the simulating results.

\section{Results}

Here, in this section it will be seen that the proposed model can explain different properties of pedestrian dynamics which were observed by conducting experiments. Experimental studies were conducted on a wide open corridor of dimension $40 \mathrm{~m} \times 2 \mathrm{~m}$, to understand the impact of corridor geometry on the pedestrian density variation. Four different types of experiments are conducted here (as indicated in Figure 5), one is on the basic corridor, and the rest three are on three different types of constrictions. In all these three cases, constriction is created longitudinally midway of the corridor. First one is by symmetrically narrowing the corridor up to a certain length; whereas, the second one by asymmetrically narrowing up to the same length (constriction in on the left side for the group of people who are moving from left to right, $L \rightarrow R$ ). The last one is a partially bifurcated corridor, created by putting an obstacle centrally. In all those four cases, experiments on unidirectional (left to right, i.e., $L \rightarrow R$ ) as well as on bi - directional flow (both from left to right and right to left, i.e. $L \rightarrow R$ and $R \rightarrow L$ ) were conducted. Density of approximately 0.6 persons $/ \mathrm{m}^{2}$ 
is used $(u-k$ diagrams indicate that at this density the flow is not in the "forced flow" regime, neither is the density very small). It must be mentioned that these four types of different pedestrian flow corridors can be observed in a lot of real life situations, like, side-walk, railway platform etc.

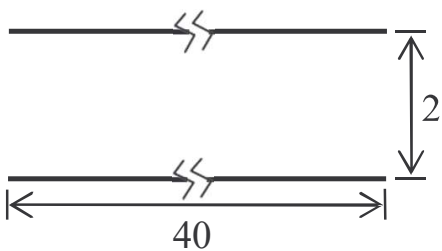

(a)

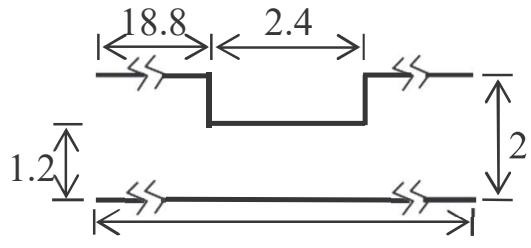

40

(c)

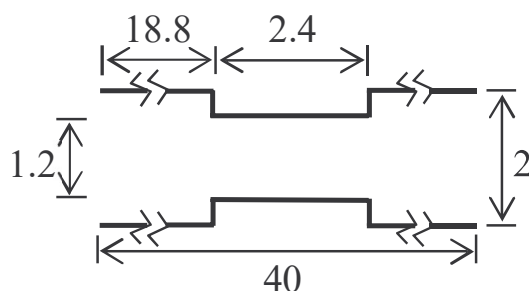

(b)

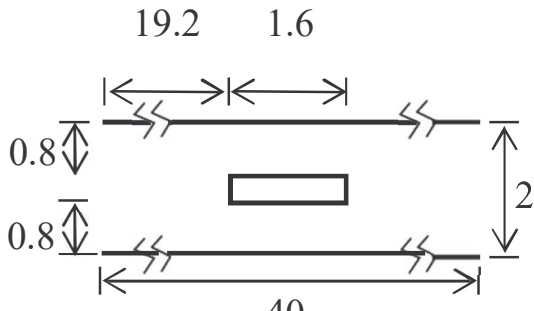

(d)

Figure 5: Schematic showing the (a) basic (b) symmetrically narrowed (c) asymmetrically narrowed and (d) partially bifurcated corridors

In the experimental set-up a corridor of $40 \mathrm{~m} \times 2 \mathrm{~m}$ with reasonable circulation area on all sides of the corridor was created by putting metal barricades (height $\approx 1.6 \mathrm{~m}$ ) on a paved surface. Experiments were conducted outdoors during daytime in pleasant weather. Two high resolution video cameras $(640 \times 480$ pixels $)$ are fitted at a height of approximately $10 \mathrm{~m}$ and a distance of $10 \mathrm{~m}$ from the near edge of the corridor and along the extended centre line of the corridor. The use of two cameras allows one to zoom in on about half the corridor length thereby allowing a good resolution during video recording. In order to determine the position of pedestrians on this corridor a grid (each cell having size $0.4 \mathrm{~m} \times 0.4 \mathrm{~m}$ ) is constructed, which is not visible to the pedestrians, but detectable in the cameras (two overhead video cameras were used to record the experiments). A tacit assumption is that a cell $(i, j)$, can never have more than one person. In order to extract meaningful information on pedestrian motion it is felt that the data need to be observed at some level of aggregation. Hence, two virtual spatial units at an aggregate level are introduced. These are:

Lane 1: This spatial unit consists of all cells $(i, j)$ for which $i=1$. In this study, five lanes (Lane 1 to Lane 5) are defined.

Zone: This spatial unit consists of all cells $(i, j)$ for which " $j$ " lies within a specified range. In this study the corridor is divided into five zones: (i) far upstream (fus) of length $12.8 \mathrm{~m}$, (ii) upstream $(u s)$ of length $6 \mathrm{~m}$, (iii) constricted $(c)$ of length $2.4 \mathrm{~m}$, (iv) downstream $(d s)$ of length 6 $\mathrm{m}$, and $(\mathrm{v})$ far downstream $(f d s)$ of length $12.8 \mathrm{~m}$. In Figure 5, those different zones are indicated through their lengths. 
It must be mentioned here that in the constricted zone, for (i) symmetrically narrowed corridor Lanes 1 and 5, (ii) asymmetrically narrowed corridor $(L \rightarrow R$ movement) Lanes 1 and 2, (iii) asymmetrically narrowed corridor ( $R \rightarrow L$ movement) Lanes 4 and 5 , and (iv) partially bifurcated corridor, Lane 3 are blocked.

In order to study the spatial variations in flow parameters the following quantities are used:

An indicator variable $O_{i, j}^{t}$ is defined as follow

$$
O_{i, j}^{t}=\left\{\begin{array}{c}
1 \text { if cell }(i, j) \text { is occupied at time } t \\
0 \text { otherwise }
\end{array}\right.
$$

Relative overall (or relative overall directional) lane density: The overall lane density for a given lane, say, Lane $1\left(O L D_{l}\right)$ is defined as the number of people occupying that lane over the entire study period. This can be obtained as:

$$
O L D_{l}=\sum_{t=1}^{T} \sum_{j=1}^{J} O_{l, j}^{t}
$$

Where $\mathrm{T}$ is the total time for which the experiment is conducted and $J$ is the maximum number of cells along the " $j$ " direction.

The relative overall lane density, $R O L D_{l}$, is defined as the fraction of people who occupied Lane $l$ to the total number of people who occupied the corridor for the entire time period. That is,

$$
R O L D_{l}=\frac{O L D_{l}}{\sum_{1=1}^{L} O L D_{l}}
$$

Where $L$ is the maximum number of lanes

A variant of this definition is useful in bi-directional flow is the relative overall directional lane density for people moving from $a$ to $b R O D L D_{l(a \rightarrow b)}$. This can be determined using the same expressions as before but by replacing $O L D_{l}$ by $O D L D_{l(a \rightarrow b)}$ and $O_{i, j}^{t}$ by $O_{i, j(a \rightarrow b)}^{t}$. 
In the foregoing part of this subsection some instances of comparison between simulated and experimental results are presented, which show that the model can explain the experimental results quite well.

Figure 6 shows the comparisons of relative overall lane density for the different "lanes" $\left(R O L D_{l}\right)$ obtained from the simulated flow with those observed from the experiments. As can be seen from the figure the predictions are quite close to the observed values.

There are similar density results for bi-directional flow also. Figure 7 shows the comparisons of relative overall directional lane density for the different "lanes" $\left(R O D L D_{l}\right)$ obtained from the simulated flow with those obtained (observed) from the experiments. In this case the relative density is calculated considering the direction in which people are moving. As can be seen from the figure the density predictions from the simulation are in reasonable agreement with the observations. As was mentioned earlier, in the asymmetrically narrowed corridor, for $L \rightarrow R$ movement, constriction is on Lanes 1 and 2; whereas, for $R \rightarrow L$ movement, it is on Lanes 4 and 5. This attributes to the differences between Figures 7(c) and 7(d).
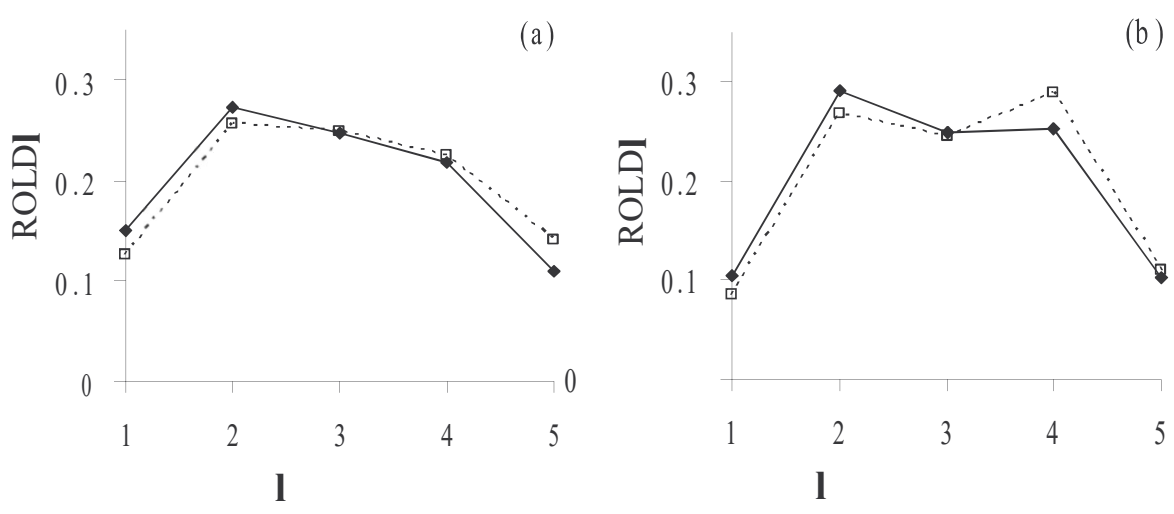

(c)

(d)
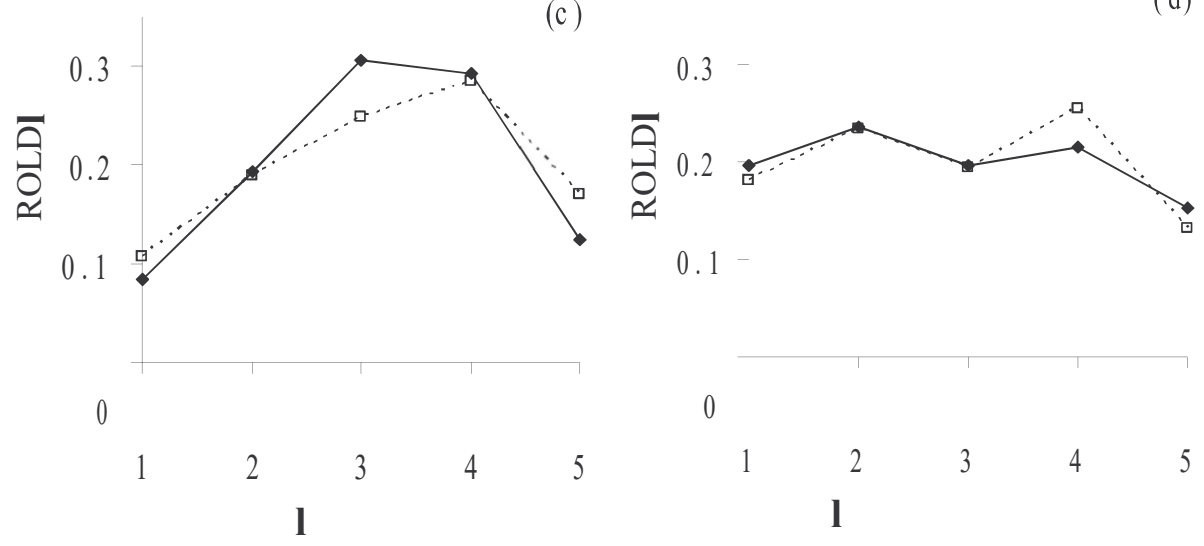

Legend:

$\longrightarrow$ Simulated densities

- - - -. Observed densities

Figure 6: Simulated and experimental $R O L D_{l}$ variations for the (a)basic (b) symmetrically narrowed (c) asymmetrically narrowed and (d) partially bifurcated corridors 

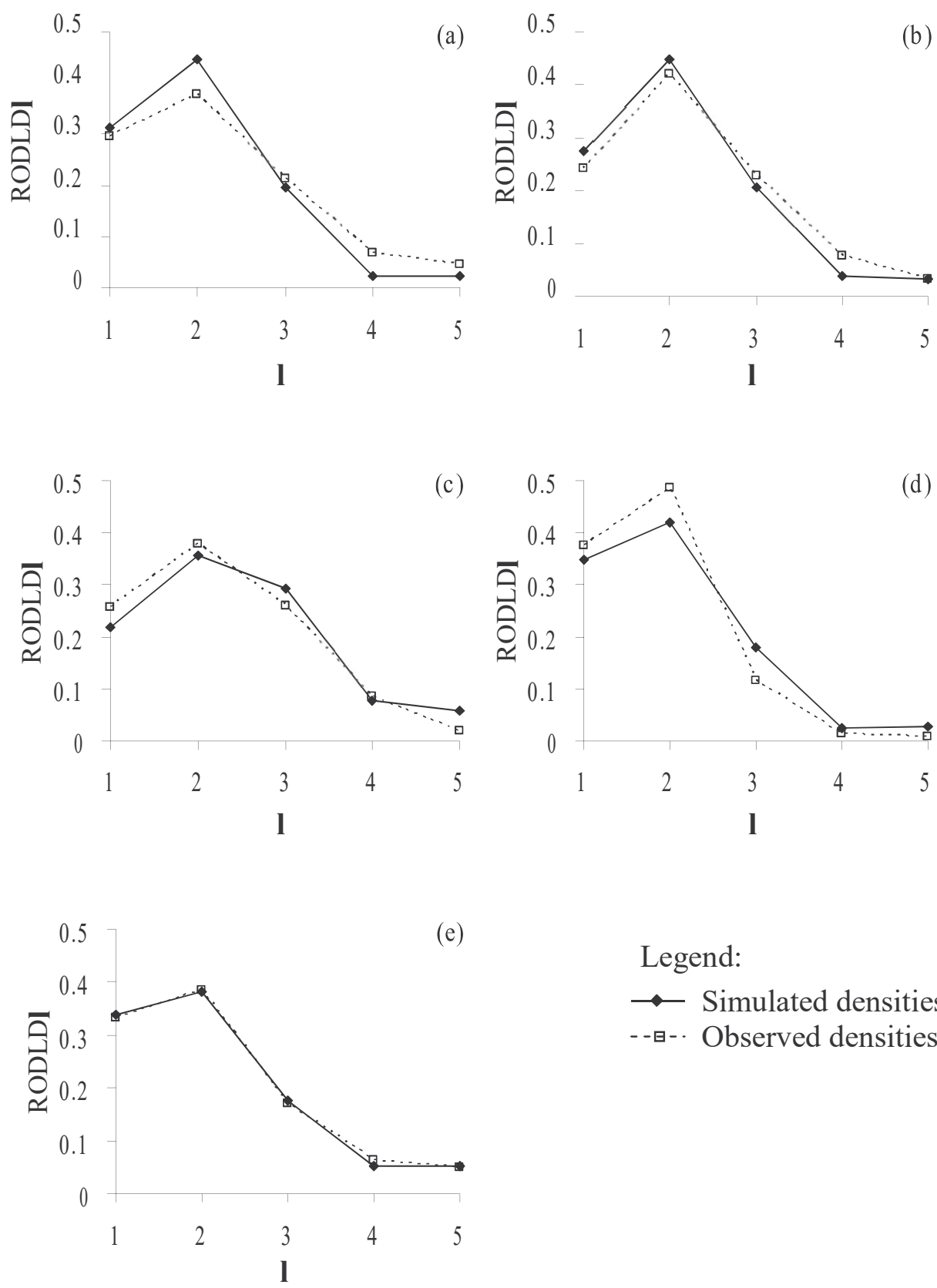

Legend:

$\rightarrow$ Simulated densities

--в-. Observed densities

Figure 7: Simulated and experimental $R O D L D_{l}$ variations for the (a) basic (b) symmetrically narrowed (c) asymmetrically narrowed (for $L \rightarrow R$ movement) (d) asymmetrically narrowed (for $R \rightarrow L$ movement) and (e) partially bifurcated corridor. 


\section{Conclusions}

The proposed discrete microscopic model is based on a fuzzy inference system, which is able to explain empirical observations for a quite large number of situations obtained from experiments. The main contribution of this work is development of FICAPeD, a fuzzy inference based microscopic model for pedestrian motion. Here in this paper pedestrian flow through corridors are explained for the sake of brevity. But it needs to be mentioned that, this calibrated and validated model can explain other pedestrian flow situations, like, single file pedestrian motion, evacuation from a hall with some entry/exits. The results from the model are promising; however, the model still requires reasonable work in terms of trying to reduce the number of empirical parameters used and to devise a better calibration strategy. This model relates to pedestrian movement in normal situations. It needs to be extended to explain pedestrian behaviour in emergency situation also.

\section{References}

Helbing D, Molnar P (1995) "Social Force Model for Pedestrian Dynamics”, Physical Review E Vol. 51 No. 5, pp. 4282-4286.

Helbing D (1991) "A Mathematical Model for the Behavior of Pedestrians", Behavioral Science Vol. 36 No. 4, pp. 298-310.

Helbing D (2000) "Simulating Dynamic Features of Escape Panic", Nature Vol. 407 No. 28, pp. 487-490.

Helbing D, Farkas IJ, Molnar P, Vicsek T (2002) "Simulation of Pedestrian Crowds in Normal and Evacuation Situation", Pedestrian and Evacuation Dynamics, Springer, Berlin, pp. 21-35.

Mehran R, Oyama A, Shah M (2009) "Abnormal Crowd Behavior Detection Using Social Force Model", Proceedings of IEEE Conference on Computer Vision and Pattern Recognition, pp. 935-942.

Song W, Xu X, Wang BH, Ni S (2006) "Simulation of Evacuation Processes Using a Multi- Grid Model for Pedestrian Dynamics", Physica A Vol. 363 No. 2, pp. 492-500.

Gruden C, Campisi T, Canale A, Tesoriere G, Sraml M (2019) “A cross-study on video data gathering and microsimulation techniques to estimate pedestrian safety level in a confined space", In IOP Conference Series: Materials Science and Engineering Vol. 603 No. 4, p. 042008.

Habib MA, Alam MJ, Holmes D (2021) "Pedestrian Micro-Simulation for Evaluating the Impacts of Social Distancing Regulations on a Dense Urban Street in Halifax, Canada", Transportation Research Board 100 th Annual Meeting No. TRBAM-2101362.

Amprasi V, Politis I, Nikiforiadis A, Basbas S (2020) "Comparing the microsimulated pedestrian level of service with the users' perception: The case of Thessaloniki, Greece, coastal front", Transportation Research Procedia 45, pp. 572-579.

Okazaki S, Matsushita S (1993) "A Study of Simulation Model for Pedestrian Movement with Evacuation and Queuing", Proceedings of International Conference on Engineering for Crowd Safety, pp. 271-281.

$\mathrm{Yu}$ WJ, Chen R, Dong LY, Dai SQ (2005) "Centrifugal Force Model for Pedestrian Dynamics", Physical Review E Vol. 72 No. 2, pp. 026112(1-7).

Blue VJ, Adler JL (1998) "Emergent Fundamental Pedestrian Flows from Cellular Automata Micro Simulation", Transportation Research Record Vol. 1644, pp. 29-36.

Blue VJ, Adler JL (1999) "Cellular Automata Microsimulation of Bi-Directional Pedestrian Flows", Transportation Research Record Vol. 1678, pp. 135-141. 
Blue VJ, Adler JL (2000) "Modeling Four Directional Pedestrian Flows", Transportation Research Record Vol. 1710, pp. 20-27.

Rothman DH, Zaleski S (1994) "Lattice-Gas Models of Phase Separation: Interfaces, Phase Transitions, and Multiphase Flow", Reviews of Modern Physics Vol. 66 No. 4, pp. 1417- 1479 .

Nagatani T (2001) "Dynamical Transition and Scaling in a Mean-Field Model of Pedestrian Flow at a Bottle-Neck", Physica A Vol. 300 No. 3, pp. 558-566.

Gipps PG, Marksjoe B (1985) "A Micro-Simulation Model for Pedestrian Flows", Mathematics and Computers in Simulation Vol. 27 No. 2-3, pp. 95-105.

Antonini G, Bierlaire M (2006) "Weber M, Discrete Choice Models of Pedestrian Walking Behavior", Transportation Research Part B Vol. 40 No. 8, pp. 667-687.

Chattaraj U, Subhashini A (2015) "Modelling Traffic Flow on Cloverleaf Interchange", European Transport Issue 58 No. 9, pp. 1-11.

Chattaraj U, Garnaik M (2015) "Effects of Highway Geometric Elements on Accident Modelling", Procedia of the Eighth International Structural Engineering and Construction Conference (ISEC-8), pp. 1171-1176. 